\title{
The Influence of Perinatal Loss on Anxiety in Multigravidas
}

\author{
By: Denise Côté-Arsenault
}

Côté-Arsenault, D. (2003). The Influence of Perinatal Loss on Anxiety in Multigravidas. Journal of Obstetric, Gynecologic, and Neonatal Nursing, 32, 623-629. DOI: $10.1177 / 0884217503257140$

\begin{abstract}
***Note: This version of the document is not the copy of record. Made available courtesy of Wiley-Blackwell. The definitive version is available at www3.interscience.wiley.com.
\end{abstract}

\begin{abstract}
:
Objective- To compare multigravid women with and without a history of perinatal loss on state anxiety, pregnancy anxiety, and optimism.

Design- Comparative descriptive; cross-sectional.

Setting- Private obstetric offices in a small northeastern city in the United States.

Participants- The sample included 160 women who were between 17 and 28 weeks gestation: 96 multigravidas with no history of loss and 74 women with a history of one or two losses.

Main Outcome Measures- State anxiety, pregnancy anxiety, optimism, and perinatal loss history. Results- No group differences were found on demographic variables, state anxiety, or optimism. However, pregnancy anxiety was higher in women with a history of perinatal loss. Pregnancy anxiety was also correlated with desire to see care provider more often and number of phone calls between visits, and was not correlated with the number of living children.

Conclusion- Women experiencing pregnancy subsequent to perinatal loss have greater pregnancy anxiety: That is, they are more concerned about their pregnancies and their babies than women without a history of perinatal loss. State anxiety and optimism do not differentiate these two groups. This heightened anxiety should be acknowledged and more frequent contact with the care provider should be offered.
\end{abstract}

\section{Article:}

Perinatal loss, defined as an undesired loss of a pregnancy through miscarriage, stillbirth, or neonatal death, occurs more frequently than generally recognized. Indeed, it has been estimated that $20 \%$ to $25 \%$ of all conceptions end unsuccessfully (Woods \& Woods, 1997). A great deal of research has focused on the experiences and consequences of perinatal loss (Frost \& Condon, 1996; Hutti, dePacheco, \& Smith, 1998). This has led to more sensitive and therapeutic nursing care following pregnancy loss through the use of protocols and the provision of support. Most women become pregnant again after a loss (Cordle \& Prettyman, 1994; Swanson, 1999), and these subsequent pregnancies are the focus of this study.

Qualitative studies of pregnancy after perinatal loss have revealed the highly anxious nature of these pregnancies (Côté-Arsenault \& Marshall, 2000; Hense, 1994; Phipps, 1985). Pregnant women have described their fear that this baby too might die. They have also reported paying 
diligent attention to every aspect of the pregnancy, wanting more frequent contact with the care provider, and being more active and directive in their own care as compared with their pregnancies prior to loss. Loss of innocence and a sense of skepticism about pregnancy and its eventual result in a live, healthy baby are common. Women no longer trust that pregnancy will end with a positive outcome (that is, a live baby) after experiencing a perinatal loss (CôtéArsenault \& Morrison-Beedy, 2001).

Anxiety in pregnancy has been a concern for several decades because of its association with negative obstetric outcomes (Lobel, 1994; Rossi, Bassi, Campanini, \& Delfino, 1991; Wadhwa, Sandman, Porto, Dunkel-Schetter, \& Garite, 1993). More recently, concern has been raised about the effect of pregnancy anxiety on parenting and the relationship of the parent with the child born subsequent to loss (Heller \& Zeanah, 1999; Theut et al., 1992). Because the methods used to define and measure anxiety have differed in many of these studies, across-study comparisons are difficult. Anxiety has been conceptualized as state anxiety (how you feel right now), trait anxiety (having a calm or anxious personality), or a more pregnancy-specific anxiety (concerns or apprehensions about this pregnancy). This study investigated the state and pregnancy-specific anxiety differences between multigravid women with and without a history of loss.

\section{Review of the Literature}

Although the effects of perinatal loss on women and families have been the subject of investigations since the early 1970s, little attention has been paid to the effects of loss on subsequent pregnancies. Phipps (1985) conducted the first known study on the subject of pregnancy after perinatal loss, using a retrospective, inductive approach with 15 couples who each had a prior neonatal death and had recently delivered a live, healthy infant. The primary findings were a heightened anxiety that was difficult for couples to manage, the expectation of a negative outcome for the pregnancy, and apparent distancing from the pregnancy.

Comparison studies of those who have a history of perinatal loss and those who do not remain limited in number. The few studies that have been conducted, beginning with Theut, Pedersen, Zaslow, and Rabinovich (1988), compared the emotional state of women (and sometimes their spouses) currently pregnant after a loss with a comparison group who were in their first pregnancies. Findings revealed that women in pregnancies subsequent to perinatal loss had more pregnancy-specific anxiety than women in their first pregnancies (Armstrong \& Hutti, 1998; Hughes, Turton, \& Evans, 1999; Theut et al., 1988). However, a more equivalent group comparison would involve women, in second or later pregnancies, with and without perinatal loss experience. It is crucial that groups have comparable obstetric history because a woman's previous pregnancy experiences influence her current pregnancy experience (Côté-Arsenault, 1999; Rubin, 1984).

Hunfeld, Agterberg, Wladimiroff, and Passchier (1996) compared a group of 24 women who had perinatal loss because of fetal anomalies detected by sonogram with a group of 26 women who had normal sonogram screenings and uneventful previous pregnancies. With this stronger comparison group design, they found that in the current pregnancies, women's anxiety prior to their sonogram screenings was higher in those with a loss history than in those without loss. In addition, women who had a live birth between the loss and their current pregnancy had less anxiety and greater quality of life than the women with no live children. However, no studies to 
date compare the anxiety of women with and without a history of perinatal loss who are in lowrisk second or later pregnancies.

Anxiety can be conceptualized as generalized or situation specific and can manifest itself in several ways during pregnancy. Overt manifestations described in the literature include a delay in telling family and friends about the pregnancy, the desire to have more frequent prenatal visits, phone calls to the care provider between scheduled visits, and requests for more prenatal testing such as sonogram screening (Côté-Arsenault \& Marshall, 2000).

Women who are in a pregnancy after perinatal loss have been described as having a "general hesitancy to look to the future” (Phipps, 1985, p. 249), as having lost their hopes and dreams, and as being depressed about previous loss. Côté-Arsenault, Bidlack, and Humm (2001) found that after loss, nearly all pregnant women in support groups included hopeful terms, along with fears and concerns, in their lists of dominant emotions. Having some hope for their pregnancies could have been a function of the support groups that perhaps cannot be generalized to other women who are pregnant after loss. In addition, not all women who have pregnancy losses become pregnant again, because they fear another loss (Cordle \& Prettyman, 1994). Optimism has not been examined in any other known study. Thus, the question arises whether women who have experienced a perinatal loss and choose to become pregnant again differ in their degree of optimism from women without a loss history.

The broad purpose of this study was to compare for state and pregnancy anxiety a group of multigravidas with a history of perinatal loss with a group of multigravidas without a history of loss. The specific research questions answered by this study were (a) Do the two groups differ on state anxiety, pregnancy anxiety, and optimism? and (b) Is state or pregnancy anxiety associated with the select prenatal variables of number of living children, desire to see the care provider more frequently, calls to care provider between scheduled visits, requests for prenatal tests, view of this pregnancy as high risk, and when they told family and friends about the pregnancy?

\section{METHODS}

A comparative descriptive design was used for this study to allow for the examination of differences between two groups of multigravid women: those who had a history of perinatal loss and those without a history of perinatal loss. A convenience sample of 170 multigravid women was recruited in a northeastern U.S. city from private obstetric practices with primarily low-risk pregnant populations. A minimum sample size of 68 in each group was predetermined to provide statistical power of .80 (Cohen, 1988).

Participants met the requirements to be included in one of two groups. For the loss group, participants were required to be (a) 18 years of age or older; (b) able to speak and read English; (c) currently pregnant, at 17 to 28 weeks gestation; (d) without a history of elective abortion; (e) classified as having a low-risk pregnancy; and (f) with a history of one or two perinatal losses (miscarriage, stillbirth, or neonatal death). The second group, to be referred to as the no loss group, met the same inclusion criteria but did not have a history of perinatal loss. A questionnaire and an information sheet were attached to the medical chart of every woman who met the inclusion criteria for either group and was currently at 17 to 28 weeks gestation. At the time of a prenatal office visit, the office staff invited women to participate and handed them the 
questionnaire and information sheet. Self-completed questionnaires were either placed into the drop box near the waiting room or mailed back in the self-addressed stamped envelopes provided. The investigator had no contact with the participants. Institutional review board approval for this study was obtained before data collection; completion of the questionnaire was construed as informed consent. A \$5 incentive was offered for participation; that incentive could be donated to the March of Dimes Birth Defects Foundation.

A gestational age of 17 to 28 weeks was chosen to ensure that all of the women would be in their 2nd trimester of pregnancy and would be physically comfortable and feeling fetal movement, but would not be focused on labor and birth. Because the majority of perinatal losses occur during the 1st trimester of pregnancy, this period of greatest concern was past for most of the women. Last, inclusion of women with low-risk pregnancies eliminated the possible confounding effect on the women's emotions that the high-risk label could have caused.

\section{Instruments}

The self-administered questionnaire, which was completed one time only, included demographics, obstetric history, Spielberger's state anxiety inventory (S-Anxiety) (Spielberger, 1983), the Pregnancy Anxiety Scale, and the Life Orientation Test (LOT).

State Anxiety. State anxiety, characterized as transitory anxiety and defined as subjective, consciously perceived feelings of apprehension and tension, was assessed using Spielberger's SAnxiety scale (1983). This 20-item, 4-point Likert-type scale is administered with the instruction, “indicate how you feel right now, at this moment.” Low scores indicate calmness and serenity; high scores reflect intense apprehension and fearfulness that approach panic. The SAnxiety has been reported as having good internal consistency (Cronbach's alpha $=.86$ to .92 ) and has been frequently used with pregnant women. Internal consistency with this sample was .94. Construct validity has been demonstrated (Spielberger, 1983).

Pregnancy Anxiety. The Pregnancy Anxiety Scale was developed for this study to tap the construct of pregnancy anxiety, defined as concern about this pregnancy and its outcome. Two items were taken with permission from the Pregnancy Outcome Questionnaire (Theut et al., 1988), an instrument intended to measure pregnancy-related anxiety. The Pregnancy Outcome Questionnaire was not used in its entirety because it appeared to tap additional constructs such as parenting anxiety, and it has never been subjected to factor analysis for support of its construct validity; therefore, only two items were adopted. Four additional items were written to tap Rubin's first two tasks of pregnancy (1984), safe passage and social acceptance (two items for each). These first two tasks were chosen because Rubin stated that if they are not undertaken successfully, then the final two tasks will not progress. A panel of experts in pregnancy (two clinical nurse specialists in maternal-child nursing and one women's health nurse practitioner) reviewed the six Pregnancy Anxiety Scale items prior to use; items were reworded based on their suggestions. A visual analogue format was used, with anchor points of Definitely Yes and Definitely No. Items were pilot-tested with 10 women, 5 with a history of perinatal loss and 5 without, for readability and appropriateness. (See Table 1 for the scale items and factor analysis.)

Factor analysis with varimax rotation revealed two factors on the Pregnancy Anxiety Scale: pregnancy concerns and concerns for the baby. The item, "I feel anxious when people talk about 
the future with this baby," did not correlate with the other items nor did it load on either factor, so it was dropped. The two-factor solution is indicative of construct validity. Additional evidence of construct validity was obtained in this study through known groups, discriminant and convergent validity analyses. The five remaining items were found to have a Cronbach's alpha of .70 in this sample, indicating adequate internal consistency for a new scale.

Table 1: Factor Loadings of the Pregnancy Anxiety Scale Items

\begin{tabular}{lll}
\hline Items & Factor 1 & Factor 2 \\
\hline When I think about this pregnancy, I feel anxious. & .78043 & .25452 \\
I feel overwhelmed because of the anxieties related to this pregnancy. & .83187 & .20255 \\
I feel confident that this baby will be fine. ${ }^{\mathrm{a}}$ & -.02671 & .86541 \\
I worry whether I will be able to bring this pregnancy to term. & .33294 & .69411 \\
I feel anxious when people talk about the future with this baby. & .79014 & -.04101 \\
I am uncomfortable talking to others about this pregnancy. ${ }^{\mathrm{b}}$ & - & - \\
Eigenvalue & 2.23468 & 1.13788 \\
\% of variance & 44.7 & 22.8 \\
\hline${ }^{\mathrm{a}}$ Requires reverse coding. & & \\
${ }^{\mathrm{b}}$ This item was dropped. & &
\end{tabular}

Optimism. The stable personality characteristic of dispositional optimism was assessed by the Life Orientation Test developed by Scheier and Carver (1985). The Life Orientation Test contains eight items and four filler items. Scores can range from 0 to 32; higher scores indicate more dispositional optimism. Four items are worded in a positive manner, and four reflect a negative or pessimistic orientation to life. Internal consistency in this sample was adequate, with a Cronbach's alpha of .85. The Life Orientation Test has been found to be valid when used with a variety of adult samples (Scheier \& Carver, 1987).

Select Prenatal Variables. Single-item, fill-in questions were asked of participants based on topics from the pregnancy literature relating to anxiety and concern during a pregnancy. These included number of living children ("fill in the number"), number of calls to the care provider between prenatal visits ("fill in the number"), desire to see the care provider more frequently (No/Yes), requests for extra prenatal tests (No/Yes), timing of telling family and friends about the pregnancy (forced choice among six options), perception of the pregnancy as high risk (No/Yes/Neither), and obstetric history of the loss(es). Obstetric history data were obtained through the completion of a pregnancy grid that asked the respondent to list each pregnancy by year, outcome, length of the pregnancy, and complications for the mother and baby.

\section{Sample}

The total sample consisted of 170 multigravidas who ranged in age from 19 to 44 years $(M=$ 32.8), were highly educated $(M=15.1$ years of education), of mid to high economic status (modal category of $\$ 40,000$ to $\$ 59,000$ reported by $28.8 \%$ ), primarily married $(92.9 \%$ ), and employed (65.3\%). Respondents were primarily White (91\%); 9\% were of varying ethnicities. Ninety-six women without a loss and 74 women with a history of loss completed the questionnaire. The respondents' obstetric history included the following: Their number of pregnancies ranged from two to six $(M=2.9)$, 82\% confirmed that they had experienced fetal movement, and $87 \%$ had at least one living child. In the loss group, participants had a mean of 1.28 perinatal losses. Their losses occurred at a mean gestational age of 10.38 weeks $(S D=6.93)$ but ranged from 2 weeks gestation to neonatal death at 2 days of age. Twenty-nine women had experienced live births before having a pregnancy loss (35.1\%), and 22 (29.7\%) had live births 
after having a pregnancy loss. The last loss occurred a mean of 2.93 years (range of 1 to 12 years) before the current pregnancy for this group.

\section{RESULTS}

\section{Group Descriptives}

Levels of state anxiety in the no loss group were moderate $(M=33.21 ; S D=10.94)$ and similar in the loss group $(M=36.27 ; S D=10.273)$. The pregnancy anxiety mean in the loss group was $40.94(S D=10.27)$ and was 31.92 in the no loss group $(S D=17.82)$. Optimism was high in both groups; the loss group mean was $29.09(S D=5.36)$, and the no loss group mean was $29.8(S D=$ 4.05).

\section{Group Comparisons}

Group comparisons were made with $t$ tests or chi square analyses. No group differences were found on demographic variables or optimism $(t=.93, p>.05)$, indicating comparability of the two groups and no group difference on optimism. State anxiety was not significantly different in the two groups $(t=-1.84 ; p=.067)$. However, the loss group had greater pregnancy anxiety than the no loss group $(t=-3.01 ; p=.003)$.

\section{Correlations}

In the total sample, Pearson product-moment correlations of the prenatal variables with pregnancy anxiety and state anxiety, respectively, revealed significant positive relationships with the perception of pregnancy as high risk, the number of calls to the care provider, and the desire to see the care provider more often. The number of living children was correlated negatively with both state and pregnancy anxiety. (See Table 2 for the correlation matrix of the study variables in the total sample.) Similar correlations were found when the loss group only was examined; however, the number of living children and the years since last loss were not significantly correlated with state or pregnancy anxiety. In addition, perception of their pregnancy as high risk was not correlated with state anxiety but was positively correlated with pregnancy anxiety. The gestational age of first loss was significantly and positively correlated with pregnancy anxiety $(r$ $=.34 ; p<.01)$ and state anxiety $(r=.41 ; p<.01)$ (see Table 3 ).

\begin{tabular}{|c|c|c|c|c|c|c|c|}
\hline Variable & 1 & 2 & 3 & 4 & 5 & 6 & 7 \\
\hline Pregnancy anxiety & - & & & & & & \\
\hline State anxiety & $.64 * *$ & - & & & & & \\
\hline Feeling of high risk & $.33^{* *}$ & $.27 * *$ & - & & & & \\
\hline Number of live children & $-.24 * *$ & $-.23^{* *}$ & -.09 & - & & & \\
\hline Optimism & $-.38^{* *}$ & $-.44 * *$ & $-.16^{*}$ & .14 & - & & \\
\hline Timing of disclosing pregnancy & .05 & .04 & .08 & -.04 & .06 & - & \\
\hline Frequency of wanting to see provider & $.26 * *$ & $.23 * *$ & $.37 * *$ & $-.20 * *$ & -.05 & $.67^{* *}$ & - \\
\hline Phone calls between visits & $.34 * *$ & $.31^{* *}$ & $.37 * *$ & $-.22 * *$ & $-.20 * *$ & -.10 & .12 \\
\hline
\end{tabular}

${ }^{*} p \geq .05 .{ }^{* *} p \geq .01$.

\section{DISCUSSION}

This comparative descriptive study used a large sample of two groups of low-risk multigravid women, at one point in pregnancy, who were equivalent demographically and obstetrically except for their history of perinatal loss. Optimism did not differ between the groups. Thus, the women pregnant after loss were no more or less optimistic than other multigravidas. This indicates that the outlook on life of the women in the two groups was similar. 
Table 3: Pearson Correlations between Main Study Variables in the Loss Group ( $\mathrm{n}=74)$

\begin{tabular}{|c|c|c|c|c|c|c|c|}
\hline Variable & 1 & 2 & 3 & 4 & 5 & 6 & 7 \\
\hline Pregnancy anxiety & - & & & & & & \\
\hline State anxiety & $.65 * *$ & - & & & & & \\
\hline Feeling of high risk & $.32^{* *}$ & .18 & - & & & & \\
\hline Number of live children & -.22 & -.18 & -.16 & - & & & \\
\hline Optimism & $-.36 * *$ & $-.42^{* *}$ & 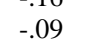 & -.08 & - & & \\
\hline Timing of disclosing pregnancy & -.11 & -.10 & -.03 & -.13 & - & - & \\
\hline Frequency of wanting to see provider & $.35^{* *}$ & $.25 * *$ & $.40^{* *}$ & -.23 & -.07 & .07 & - \\
\hline Phone calls between visits & $.32 * *$ & $.27 * *$ & .21 & -.19 & -.25 & -.10 & .11 \\
\hline
\end{tabular}

${ }^{*} p \geq .05 .{ }^{* *} p \geq .01$.

What did differentiate these groups emotionally was pregnancy anxiety, identified with the Pregnancy Anxiety Scale, which was found to have adequate reliability and validity. This finding of pregnancy-specific anxiety is perhaps the most noteworthy. Indeed, women who had previous unsuccessful pregnancies were more anxious about their pregnancies and babies than were women who had only successful previous pregnancies. Therefore, it is important that pregnancy anxiety be measured in future studies, not simply state or generalized anxiety, because these generalized measures do not address the specific concerns of women in pregnancy after loss.

The findings of this study, higher pregnancy-related anxiety in those pregnant after loss, corroborate the findings of Theut et al. (1988). This study had greater internal validity, however, due to the comparison of multigravidas who had experienced perinatal loss with multigravidas who had not had such loss. The Pregnancy Anxiety Scale also was found to be psychometrically sound, with adequate reliability and evidence of validity (Côté-Arsenault, 1999). Findings in this study are also congruent with those of Hunfeld et al. (1996), but with a low-risk, larger sample.

Having other living children was not significantly associated with lower pregnancy anxiety. This verifies that the previous experience of perinatal loss remains with a woman, even if she has other children, and corroborates similar qualitative findings (Côté-Arsenault \& Morrison-Beedy, 2001). Hunfeld et al. (1996) found that giving birth to a live baby between the loss and the current pregnancy did affect the amount of pregnancy-related anxiety when measured with the Pregnancy Outcome Questionnaire.

Gestational ages at the time of loss in this sample were primarily in the 1st trimester, thus most of the women in the loss group were past the time of their previous perinatal losses. Despite this, higher anxiety was found in the loss group. This finding contradicts two common assumptions: first, that a 1st trimester miscarriage is not significant or important (Layne, 1990), and second, that once they get past the point of a previous loss, such women are fine (see Côté-Arsenault \& Dombeck, 2001, for further details). It would be reasonable to anticipate even higher pregnancy anxiety when the pregnancy is designated as high risk and when previous loss occurred late in the pregnancy.

\section{Clinical Implications}

Those with higher anxiety (pregnancy and state) wanted more frequent contact with their health care providers, both through phone calls and through increased frequency of visits. Offering these supportive services to prenatal patients would validate their anxiety and demonstrate the availability of support whenever it is needed. Indeed, phone calls and drop-in prenatal visits to check on the fetal heart rate should be encouraged if they are useful coping strategies for families 
with high anxiety. These women are seeking outside, objective data that their babies are developing normally, at least at this moment (Côté-Arsenault \& Marshall, 2000). Although this reassurance is temporary, women and their families will recognize that they can receive such support throughout their pregnancies. Referral to support groups, when available, may also be appropriate. Such groups have been found to be effective in helping families deal with issues surrounding pregnancies after loss and cope better with their anxieties and fears (Côté-Arsenault \& Freije, 2002).

\section{Research Implications}

This study was limited by its cross-sectional design and convenience sampling. The largely homogeneous sample of primarily White, well-educated, and upper-income women also limits the generalizability of the findings. Qualitative studies have identified that anxiety goes up and down in pregnancies after loss, indicating the need for a prospective study. A longitudinal study could shed some light on what occurs across the three trimesters, rather than taking a snapshot at one time in pregnancy. Interventions that would be helpful to these families throughout their pregnancies and perhaps afterward need to be developed and empirically tested. Lack of evidence-based protocols for the specialized care of women and their families in pregnancies after perinatal loss is a major void in the care that we provide.

\section{ACKNOWLEDGMENTS}

The author acknowledges the funding support of the National Institute of Nursing Research (1 F31 NR06778-01) for this study and the assistance of Noreen Salo, MS, RNC, with data collection and entry.

\section{REFERENCES}

Armstrong, D., \& Hutti, M. A. (1998). Pregnancy after perinatal loss: The relationship between anxiety and prenatal attachment. Journal of Obstetric, Gynecologic, and Neonatal Nursing, 27, 183-189.

Cohen, J. (1988). Statistical power analysis for the behavioral sciences (2nd ed.). Hillsdale, NJ: Lawrence Erlbaum.

Cordle, C. J., \& Prettyman, R. J. (1994). A two year follow-up of women who have experienced early miscarriage. Journal of Reproductive and Infant Psychology, 12, 37-43.

Côté-Arsenault, D. (1999). Pregnancy after loss [Letter to the editor]. Journal of Obstetric, Gynecologic, and Neonatal Nursing, 28, 13-14.

Côté-Arsenault, D., Bidlack, D., \& Humm, A. (2001). Women’s emotions and concerns during pregnancy following perinatal loss. Journal of Nursing Scholarship, 33, 128-134.

Côté-Arsenault, D., \& Dombeck, M. T. (2001). Maternal assignment of fetal personhood to a previous pregnancy loss: Relationship to anxiety in the current pregnancy. Health Care for Women International, 22, 649-665. 
Côté-Arsenault, D., \& Freije, M. M. (2002). Making it through bittersweet pregnancies in a safe place: A focused ethnography. Manuscript submitted for publication.

Côté-Arsenault, D., \& Marshall, R. (2000). One foot in—one foot out: Weathering the storm of pregnancy after perinatal loss. Research in Nursing and Health, 23, 473-485.

Côté-Arsenault, D., \& Morrison-Beedy, D. (2001). Women’s voices: Reflecting back on pregnancy after perinatal loss. Journal of Nursing Scholarship, 33(3), 239-244.

Frost, M., \& Condon, J. T. (1996). The psychological sequelae of miscarriage: A critical review of the literature. Australian and New Zealand Journal of Psychiatry, 30, 54-62.

Heller, S. S., \& Zeanah, C. H. (1999). Attachment disturbances in infants born subsequent to perinatal loss: A pilot study. Infant Mental Health Journal, 20(2), 188-199.

Hense, A. L. (1994). Livebirth following stillbirth. In P. A. Field \& P. B. Marck (Eds.), Uncertain motherhood: Negotiating the risks of the childbearing years (pp. 163-194). Thousand Oaks, CA: Sage.

Hughes, P. M., Turton, P., \& Evans, C. D. H. (1999). Stillbirth as a risk factor for depression and anxiety in the subsequent pregnancy: Cohort study. British Medical Journal, 318, 17211724.

Hunfeld, J. A. M., Agterberg, G., Wladimiroff, J. W., \& Passchier, J. (1996). Quality of life and anxiety in pregnancies after late pregnancy loss: A case-control study. Prenatal Diagnosis, 16, 783-790.

Hutti, M. H., dePacheco, M., \& Smith, M. (1998). A study of miscarriage: Development and validation of the Perinatal Grief Intensity Scale. Journal of Obstetric, Gynecologic, and Neonatal Nursing, 27, 547-555.

Layne, L. L. (1990). Motherhood lost: Cultural dimensions of miscarriage and stillbirth in America. Women \& Health, 16, 69-98.

Lobel, M. (1994). Conceptualizations, measurement and effects of prenatal maternal stress on birth outcomes. Journal of Behavioral Medicine, 17(3), 225-272.

Phipps, S. (1985). The subsequent pregnancy after stillbirth: Anticipatory parenthood in the face of uncertainty. International Journal of Psychiatry in Medicine, 15(3), 243-264.

Rossi, N., Bassi, L., Campanini, D., \& Delfino, M. D. (1991). Maternal anxiety and childbirth. Clinical and Experimental Obstetrics \& Gynecology, 18(1), 19-25.

Rubin, R. (1984). Maternity identity and the maternal experience. New York: Springer. 
Scheier, M. F., \& Carver, C. S. (1985). Optimism, coping and health: Assessment and implications of generalized outcome expectancies. Health Psychology, 4(3), 219-247.

Scheier, M. F., \& Carver, C. S. (1987). Dispositional optimism and physical well-being: The influence of generalized outcome expectancies on health. Journal of Personality, 55(2), $169-210$.

Spielberger, C. D. (1983). Manual for the state-trait anxiety inventory. Palo Alto, CA: Consulting Psychologists Press.

Swanson, K. M. (1999). Effect of caring, measurement, and time on miscarriage impact and women’s well-being. Nursing Research, 48, 288-298.

Theut, S. K., Moss, H. A., Zaslow, M. J., Rabinovich, B. A., Levin, L., \& Bartko, J. J. (1992). Perinatal loss and maternal attitudes toward the subsequent child. Infant Mental Health Journal, 13(2), 157-166.

Theut, S. K., Pedersen, F. A., Zaslow, M. J., \& Rabinovich, B. A. (1988). Pregnancy subsequent to perinatal loss: Parental anxiety and depression. Journal of the American Academy of Child and Adolescent Psychiatry, 27(3), 289-292.

Wadhwa, P. D., Sandman, C. A., Porto, M., Dunkel-Schetter, C., \& Garite, T. J. (1993). The association between prenatal stress and infant birth weight and gestational age at birth: A prospective investigation. American Journal of Obstetrics and Gynecology, 169, 858865.

Woods, J. R., \& Woods, J. L. (Eds.). (1997). Loss during pregnancy or in the newborn period. Pittman, NJ: Jannetti. 